\title{
TITLE: Between GERD and NERD: The Relevance of Weakly Acidic Reflux
}

AUTHORS: Nicola de Bortoli ${ }^{1}$, Andrea Ottonello ${ }^{2}$, Frank Zerbib ${ }^{3}$, Daniel Sifrim ${ }^{4}$, C. Prakash Gyawali $^{5}$, Edoardo Savarino ${ }^{2}$

\section{AFFILIATIONS:}

${ }^{1}$ Division of Gastroenterology, Department of Translational Research and New Technology in Medicine and Surgery, University of Pisa, Pisa, Italy

${ }^{2}$ Division of Gastroenterology, Department of Surgery, Oncology and Gastroenterology, University of Padua, Padua, Italy

${ }^{3}$ Department of Gastroenetrology, CHU Bordeaux and Bordeaux University, Bordeaux, France

${ }^{4}$ Barts and the London School of Medicine and Dentistry, Queen Mary University of London, UK

${ }^{5}$ Division of Gastroenterology, Washington University School of Medicine, St. Louis, Missouri

\section{Authors contribution:}

- Nicola de Bortoli, MD, PhD: data collection and analysis, writing of the manuscript, approving final version

- Andrea Ottonello, MD, PhD: data collection and analysis, writing of the manuscript, approving final version

- Frank Zerbib, MD: data collection and analysis, approving final version

- Daniel Sifrim, MD, PhD: data collection and analysis, approving final version

- C. Prakash Gyawali, MD: writing of the manuscript, approving final version

- Edoardo Savarino, MD: data collection and analysis, approving final version

Short title: Weakly acidic Reflux in GERD

Keywords: heartburn; multichannel impedance and $\mathrm{pH}$ monitoring; proton pump inhibitors; GERD; NERD; weakly acidic reflux 


\section{Corresponding Author:}

Edoardo V. Savarino

Division of Gastroenterology, Department of Surgery, Oncology and Gastroenterology

University of Padua

Via Giustiniani 2

35128 Padova

Italy

Telephone: +390498217749;

Fax: +390498760820 ;

e-mail: edoardo.savarino@unipd.it 


\section{ABSTRACT (Word-count 192)}

Non-erosive reflux disease (NERD) is a common condition and the most frequent phenotype of GERD. NERD is extremely heterogeneous and includes patients with negative endoscopy, but abnormal esophageal acid exposure and/or positive reflux-symptom association analysis (hypersensitive esophagus). This segregation is only possible thanks to the use of impedance-pH monitoring. Indeed, weakly acidic reflux represents one of the most frequent cause of refractory symptoms in patients evaluated off-anti-secretory therapy and, more importantly, during antisecretory drug treatment. Patients with heartburn who do not have any type of reflux underlying their symptoms (functional heartburn) must be excluded from the category of GERD. The drawbacks of impedance-pH are mainly due to the day-to-day variability of the test and by the fact that the accuracy of the symptom-reflux correlation scores is often far from being perfect. Some histopathological characteristics, as dilated intercellular spaces, can be helpful to distinguish patients with NERD by esophageal biopsies. As to the outcome, patients with NERD in whom acid is the main pathogenetic factor respond successfully to PPI therapy, while those with hypersensitive esophagus to weakly acidic reflux could be treated with reflux inhibitors or surgery, although further controlled studies are required. 


\section{Definition of NERD and sub-groups}

Heartburn, or retrosternal burning, is a typical symptom of gastroesophageal reflux disease (GERD) that frequently affects a large part of population (10\%-20\%) in Western countries, interfering significantly with the quality of life of many patients. ${ }^{1}$

For many years, erosive reflux disease has been considered the more common manifestation of GERD, nevertheless, in the past decade it has been realized that erosive reflux disease represents the minority of patients with GERD $(\sim 30 \%)$, whereas the majority of them $(\sim 70 \%)$ are included in the non-erosive reflux disease (NERD) phenotype, characterized by the presence of typical reflux symptoms without any esophageal mucosal lesion visible at endoscopy. ${ }^{2,3}$

Pathophysiological studies carried out with 24-hour esophageal multichannel intraluminal impedance-pH testing (MII-pH) have demonstrated that patients with NERD phenotype are markedly heterogeneous and can be subdivided into several well-defined subgroups (after excluding eosinophilic esophagitis, EoE and primary esophageal motor disorders). Currently, the NERD definition includes patients with negative endoscopy but abnormal esophageal acid exposure $(\mathrm{AET}) .^{3-7}$

Further, in the NERD population, we can include patients with negative endoscopy and normal pathophysiological testing (pH-metry/MII-pH), both for AET and total number of reflux events, but with these latter variables temporarily correlated with symptoms (Hypersensitive esophagus, HE). ${ }^{7}$ The correlation between symptoms and reflux events is commonly defined by means of symptom index (SI positive if $>50 \%$ ) and symptom association probability (SAP, positive if > 95\%). ${ }^{6}$ Patients may be hypersensitive to both acid and/or non-acid reflux events. ${ }^{4,8,9}$ Patients with heartburn refractory to proton pump inhibitors (PPIs), who show negative endoscopy, normal AET and number of refluxes and no correlation between symptoms and reflux, should be considered as patients with functional heartburn $(\mathrm{FH})^{4,6,7,10}$ and should not be considered and treated as GERD patients (i.e. stopping any kind of anti-secretory therapy and not undergoing antireflux surgery). Accordingly, the last iteration of Rome criteria defined FH as "chronic retrosternal 
burning, discomfort or pain refractory to optimal antisecretory therapy in the absence of GERD, histopathologic mucosal abnormalities, major motor disorders, or structural explanations. ${ }^{7}$ In line with this definition, Savarino et al. ${ }^{10}$ demonstrated the added value of MII-pH in distinguishing patients with GERD from those affected by FH, by showing a $10 \%$ diagnostic gain with this technique. Similar results were obtained in some studies performed both "off PPI therapy" ${ }^{\text {, 11-14 }}$ (10-15\% diagnostic gain) and "on PPI therapy"11, 12, 15, 16 (20-40\% diagnostic gain) with MII-pH testing. Overall, the added values and the clinical application of impedance monitoring should help the clinicians to correlate an increased number of symptoms with reflux events, thus increasing the number of patients with reflux disease and reducing the rate of patients with $\mathrm{FH}$.

\section{Clinical characteristics}

The positive or negative response of patients with heartburn to PPI therapy is an empiric criterion, which can include or exclude from the definition of GERD those patients who complain of heartburn and do not have an objective demonstration of the presence or absence of gastroesophageal reflux. However, it has been estimated that $25 \%-40 \%$ of patients complaining of heartburn continue to present symptoms despite daily PPI use and that there is a large group of patients with functional disorders rather than GERD who may respond to PPI due to a placebo effect. ${ }^{5,17}$ Previous studies have underlined this important limitation, which affects the diagnostic accuracy of the PPI test. ${ }^{18,19}$ In particular, Bytzer et al. ${ }^{20}$ carried out a study in a sample of welldefined primary care patients with suspected GERD, and demonstrated the limited ability of a PPI trial to identify patients with GERD, using endoscopy, esophageal $\mathrm{pH}$-metry and reflux disease questionnaires as reference standard. Presence or absence of gastro-esophageal reflux during barium esophagography does not correlate with incidence or extent of reflux observed during MII$\mathrm{pH}$ monitoring and it is not of value for the diagnosis of GERD. ${ }^{19,21}$

A symptom-based approach using a questionnaire (GERDQ) does not always confirm GERD diagnosis. ${ }^{22,23}$ Multiple studies have demonstrated that patients with objective evidence of 
reflux tend to be older, male, and smokers in comparison to patients with $\mathrm{FH} .{ }^{24-27}$ Increased body mass index $\left(>25 \mathrm{~kg} / \mathrm{m}^{2}\right)$ is also associated with PPI failure in patients with acid reflux compared with those patients with $\mathrm{HE}$ or $\mathrm{FH} .{ }^{27-29}$ Further, there are several evidences suggesting a strong association between FH and other functional disorders such as functional dyspepsia and/or irritable bowel syndrome, emphasizing the hypothesis of a unique "whole gut" GI disorder. ${ }^{30-32}$

However, currently, demographics do not reliably distinguish subgroups of patients suffering from heartburn. ${ }^{25,33}$ Understanding the epidemiology and pathogenesis of patients with FH may allow early recognition of these patients and could help to anticipate and avert therapeutic failure. ${ }^{34}$

\section{MII-pH diagnosis and different GERD phenotypes}

Patients often undergo extensive functional testing to evaluate heartburn if there is inadequate response to optimal acid suppressive therapy, as this approach seems to be useful in differentiating FH from refractory acid reflux. To note, optimal acid therapy is not well-defined in the literature. In the setting of objective acid testing, an effective therapy may be considered the adequate control of acid assessed by a functional examination. However, a simple and more often used definition for optimal acid suppression is the lack of symptoms in response to acid suppression (defined as twice a day proton pump inhibition). ${ }^{6,35}$

The complete chemical nature of reflux can be detected neither by traditional esophageal pH-metry nor by the catheter-free Bravo $^{\mathrm{TM}}$ system, ${ }^{36}$ but the advent of $24 \mathrm{~h}$ MII-pH testing has enabled differentiation of acid (reflux with $\mathrm{pH}<4$ ) from weakly acidic $(4<\mathrm{pH}<7)$ or weakly alkaline reflux $(\mathrm{pH}>7) .{ }^{37}$ Indeed, reflux of gastric content into the esophagus is a physical event that has been evaluated for long time only by means of techniques able to obtain information on the chemical component of refluxate. The MII-pH was an innovative technique that provided a detailed characterization of each reflux event including chemical (acid, weakly acidic/alkaline reflux) and physical properties (liquid, mixed, gas). ${ }^{37,} 38$ In the last decade, it has been shown that nonacid 
reflux (weakly acidic and weakly alkaline reflux events) represents the majority of reflux episodes in patients with GERD on PPI therapy. ${ }^{39,}{ }^{40}$ Indeed, the total number of reflux episodes is not affected by acid suppressive therapy, and weakly acidic reflux accounts for approximately $90 \%$ of all reflux episodes in patients on PPIs, thus representing a potential mechanism underlying the failure of PPI treatment in patients with reflux-related symptoms. ${ }^{12,15}$ Further, the assessment of both acid and non-acid reflux correlation with symptoms may allow us to better select patients who would benefit from anti-reflux surgery or an endoscopic anti-reflux procedure. ${ }^{41}$

However, all of the available tests for GERD diagnosis have some limitations. The drawbacks of MII-pH are mainly due to the day-to-day variability of the test. ${ }^{42-44}$ Additionally, the reflux-symptom correlation in patients with GERD who do not respond to PPI therapy is actually calculated by the symptom index (SI) or symptom association probability (SAP), although their validity is still uncertain. ${ }^{45,46}$ Unfortunately, symptoms may not occur during 24-hour reflux monitoring. Moreover, the accuracy of patients in symptom recording is often far from perfect. As a consequence, SAP/SI may be negative even in several patients with erosive reflux disease (ERD). ${ }^{16 \text {, }}$ 47-49 Therefore, in pH-negative patients a positive SAP/SI indicates reflux-related heartburn, ${ }^{40}$ but a negative SAP/SI does not exclude GERD; indeed, patients with heartburn may respond to PPI therapy despite normal MII-pH findings. ${ }^{51}$ Accordingly, Zerbib et al. ${ }^{52}$ reported that MII-pH results were not always able to predict the response to PPIs in patients with typical reflux-related symptoms, when the test was performed off PPI therapy.

Recently, the ability of MII-pH testing in better understanding GERD pathophysiology has improved by means of new parameters, such as the post-reflux swallow-induced peristaltic wave (PSPW) index, which indicates the efficacy of esophageal clearance, ${ }^{47}$ and the baseline impedance values, which indicate lack of integrity in the esophageal mucosa. ${ }^{16,39,47,53,54}$ PSPW index has been shown to be lower in patients with abnormal AET, compared to healthy volunteers (HVs) or FH. ${ }^{47}$ Moreover, this parameter was not altered after medical or surgical therapy. ${ }^{55}$ Further, Kessing et al. ${ }^{55}$ described lower values of baseline impedance levels in the distal esophagus of patients with 
abnormal esophageal AET, compared to HVs. Recently, many studies standardized the measurement of baseline impedance by calculating the mean nocturnal baseline impedance (MNBI) across three 10-min periods between $1 \mathrm{AM}$ and $3 \mathrm{AM}$, away from daytime esophageal physiologic activity. ${ }^{48,51,56}$ The most relevant advantages regarding both the MBNI and PSPW index have been recently published by Frazzoni et al. ${ }^{48}$ The authors showed a gradient behaviour of them across 289 GERD patients and 50 healthy controls, being both parameters worse in erosive esophagitis compared with NERD and healthy controls. The diagnostic yield of both parameters was greater than that of increased AET, total reflux events, and bolus exposure time in both erosive esophagitis and NERD. Furthermore, MNBI and PSPW index make pathophysiologic sense, and certainly deserve a chance in redeeming the clinical value of ambulatory MII-pH testing. ${ }^{57}$

There are still others information that could be caught from a more in deep analysis of MII$\mathrm{pH}$ tracing such as the role of bolus contact time in improving GERD diagnosis ${ }^{58}$ or gas and mixed reflux or supragastric belching in increasing symptom perception. ${ }^{59,60}$ Further, the role of this technique in the management of patients with extraesophageal GERD has been partially investigated. ${ }^{61-67}$

\section{Weakly acidic reflux and refractory heartburn}

The Porto consensus report provided a detailed nomenclature for reflux patterns detected by MII- $\mathrm{pH}$ monitoring. ${ }^{37}$ An impedance-detected reflux is defined as acid when the esophageal $\mathrm{pH}$ falls to $<4$, or when reflux occurs with the esophageal $\mathrm{pH}$ already $<4$. When the esophageal $\mathrm{pH}$ falls by $\geq 1$ unit, but remains $>4$, it is considered "weakly acidic reflux."

The prevalence of weakly acidic reflux (WARs) in refractory GERD depends on the way reflux monitoring is performed. Although several WARs episodes can be detected during 24-hour MII-pH monitoring “off” PPI, this type of refluxate becomes particularly significant during studies "on" PPI. ${ }^{12,15,68,69}$ First of all, Vela et al. ${ }^{69}$ used stationary MII-pH monitoring to compare 3-h postprandial recordings of the same subject "on" and "off" PPI. The antisecretory treatment 
provoked no reduction in the total number of reflux events, but there was a shift in the refluxate's pH from acidic to weakly acidic. Heartburn was replaced by regurgitation, which became the predominant symptom in these patients. Similarly and by means of the more reliable 24-hour $\mathrm{pH}$ impedance monitoring, Frazzoni and co-workers showed that WARs are the predominant reflux events in patients evaluated on-PPI. ${ }^{16,39,54}$

Studies in patients with refractory GERD showed that WARs could be associated with $30 \%$ $40 \%$ of symptoms. ${ }^{12,15} 16,54$ In another study Zerbib et al, ${ }^{70}$ observed that in a group of patients with refractory heartburn WARs were associated to both heartburn and regurgitation in patients evaluated on double dose PPI therapy. The role of WARs in patients with typical refractory symptoms has been studied, almost always, in patients on PPIs. Few but intriguing data have been reported on WARs evaluated in patients off-therapy and it was confirmed that this type of chemical reflux can be associated with both heartburn and regurgitation. ${ }^{49,71,72}$

A prospective study in well selected PPI responder and non-responder NERD patients, in whom MII-pH was performed both off an on-PPIs, ${ }^{73}$ showed that WAR accounted for approximately $30 \%$ of symptomatic refluxes both in non-responder and in responder patients whereas an overall increased number of reflux episodes and an enhanced sensitivity to all episodes could predict treatment failure. Comparable finding were reported in consecutive refractory patients - 50\% of them affected by erosive esophagitis - undergone laparoscopic fundoplication ${ }^{16}$ and repeated MII-pH monitoring performed on PPIs before treatment and off PPIs at follow up. In that series, the 3-year outcome assessment revealed that decrease of the abnormal number of all reflux episodes (also due to a decrease of WARs), normalization of acid exposure and of SAP/SI were associated with a sustained symptom remission.

The mechanisms by which WARs can provoke persistence of symptoms remain controversial, as several factors have been proposed: (1) esophageal distension by increased reflux volume ${ }^{70}(2)$ persistent impairment of esophageal mucosa due to weakly acidic reflux containing bile acids, ${ }^{74}$ (3) the proteolytic activity of pepsins that is maintained up to $\mathrm{pH} 6^{75}$ and healing of 
mucosal breaks occurs through reparative processes that are inhibited at $\mathrm{pH} 6.5$ and abolished at $\mathrm{pH}$ $3.0{ }^{75}$ (4) esophageal hypersensitivity to non-acid components of gastric contents either when gas is present in the refluxate or after esophageal sensitization due to an acid reflux ${ }^{76,77}$ and (5) reduced esophageal chemical clearance. ${ }^{78}$ Once refluxate has entered the esophagus the main defense against persistence of mucosal damage is removal of the noxious agents as quickly as possible: indeed, defective chemical clearance of WARs, which represents the vast majority of reflux events in PPI-refractory GERD, has a key role in the pathogenesis of PPI-refractory reflux esophagitis. ${ }^{47,78}$

By the way, there is no doubt that WARs can be one of the underlying mechanisms of refractory GERD. Indeed, a cause-and-effect relationship between WARs and PPI-refractory heartburn/regurgitation has been shown in some prospective observational studies addressing post surgical outcome in PPI-refractory typical GERD. ${ }^{16,54}$ These studies well evidenced that WARs might be considered the major determinant of typical GERD-related symptoms in PPI-refractory patients. Anyway, future prospective studies should be addressed to confirm these data in a larger series of patients.

\section{Histopathology changes and mucosal barrier integrity in NERD subgroups}

The integrity of the mucosal epithelial barrier is of great importance to prevent pathologic consequences of reflux, and can be overcome in the disease state (whether erosive or non-erosive). So, it is of relevance to discuss the structures responsible for maintenance of mucosal integrity.

The pre-epithelial defense consists of a small water layer with limited buffering capacity, presumably due to the presence of bicarbonate derived from swallowed salivary fluid and from secretions of esophageal submucosal glands. ${ }^{79}$ In patients with esophagitis there is a clear breach in

this barrier allowing components of the refluxate to reach the nociceptors in the lamina propria. ${ }^{79}$ Acid and acid-pepsin initially attack and damage the intercellular junctions, thus resulting in an increase in para-cellular permeability, reflected morphologically by the presence of dilated intercellular spaces. ${ }^{80}$ 
From a diagnostic point of view, esophageal biopsies might be helpful to identify patients with histological signs of GERD. Further, it is relevant to underline that the addition of esophageal biopsies as an adjunct to an endoscopic examination has been re-emphasized because of the progressively increased detection of eosinophilic esophagitis (EoE). Many clinicians routinely take esophageal biopsies in patients with reflux-type symptoms to search for EoE in the setting of an endoscopy that does not reveal erosive changes. ${ }^{81}$

Histological examination of esophageal biopsies may help in distinguishing patients with NERD from $\mathrm{FH}$, as dilated intercellular spaces can be a microscopic marker of reflux and esophageal damage frequently associated with NERD and only rarely with FH. ${ }^{82}$ The presence of dilated intercellular spaces may also predict a non-response to acid suppression. ${ }^{83}$ Using light microscopy (LM), it is possible to combine multiple histological alterations denoting the presence of microscopic esophagitis (ME), such as basal cell hyperplasia, papillae elongation, and DIS. ${ }^{84-86}$

Zentilin et al. ${ }^{85}$ proposed a histological score able to discriminate between GERD and controls with a positive predictive value of $97 \%$ and a negative predictive value of $46 \%$.

It has been also hypothesized that there is a good correlation between DIS in the esophageal epithelium of both ERD and NERD patients and the presence of heartburn. ${ }^{87}$ Recently, Savarino et al. ${ }^{88}$ demonstrated the lack of microscopic esophagitis (ME) in the esophageal distal biopsies of FH patients, suggesting a limited role of these histological abnormalities in symptom generation in them. ME can be considered as an accurate and reliable diagnostic marker for distinguishing FH patients from GERD patients and has the potential to be used to guide the correct therapy.

Fiocca et al. ${ }^{89}$ developed consensus guidelines for histologic recognition of ME in patients with GERD and proposed several criteria, which achieved high levels of agreement when assessed independently by 5 pathologists.

Recently, Kandulski et al..$^{90}$ confirmed that esophageal biopsies are useful to differentiate NERD from FH, especially in patient with refractory heartburn. The same authors showed that low levels of baseline impedance (detected with 24-h MII-pH) are associated with increased exposure to 
acid and dilation of intercellular spaces, indicating a strong correlation between esophageal mucosal impairment and baseline impedance. ${ }^{91}$

However, these histological alterations are not still adequately sensitive and specific to be used in a diagnostic algorithm, and so far routine esophageal biopsies as a means of making a sound diagnosis of $\mathrm{FH}$ are not recommended.

\section{Aerophagia, gas reflux and supragastric belching in pathophysiology of NERD}

Aerophagia is a condition of excessive air swallowing, which goes to the stomach. Pouderoux et al. $^{92}$ observed, by means of ultrafast computerized tomography, a substantial aerophagia (8-32mL of air) during transit of a swallowed bolus through the esophagus and a partial bolus separation with air preceding fluid. Bravi et al. ${ }^{93}$ demonstrated that PPI non-responder patients with GERD swallowed more air at mealtime than those who respond to PPI treatment and also have more reflux episodes that contain gas. The authors concluded that air swallow combined with mucosal sensitization could affect perception of symptoms.

Gastric belching (frequent gas-reflux events during MII-pH 24-h) is the escape of swallowed intragastric air that enters the esophagus during a transient lower-esophageal sphincter relaxation (TLESR). ${ }^{94}$ Gastric belches occur 25 to 30 times per day and are physiological, involuntary and controlled entirely by reflexes. Belching does not seem to facilitate acid reflux in healthy subject. ${ }^{95}$ On the other hand, the presence of gas into the refluxate enhances reflux perception, is frequently associated with proximal extent of reflux and occurs more frequently in patients who do not respond to acid suppressive treatment. ${ }^{71,77,93,96}$

In supragastric belches the air does not originate from the stomach but is ingested immediately before it is expelled again. ${ }^{97}$

Supragastric belches are not a reflex but, instead, are the result of human behavior. Studies with simultaneous impedance monitoring and high-resolution manometry reveal the underlying mechanism of this behavior: a contraction of the diaphragm creates a negative pressure in the 
thoracic cavity and the esophagus, subsequent relaxation of the UES, resulting in inflow of air into the esophagus. ${ }^{98}$ It is unclear what causes supragastric belching and what causes patients to start this behavior. Some patients report that initially they belched purposefully to relieve a sensation of bloating or abdominal discomfort but that with time they lost control of the belching. Many patients stop belching during speaking and sleeping; it has been shown that distraction also reduces the frequency of belching, ${ }^{97}$ whereas putting attention to their belching behavior usually results in an increase in belching frequency. ${ }^{97}$ Recently, Koukias et al. ${ }^{60}$ described 100/2950 patients, over a 4 years period, with supragastric belching that were associated more frequently with pathological acid exposure and esophageal hypomotility.

Speech therapy has been proposed in a recent pilot study including 11 patients and resulted to be beneficial. ${ }^{99}$ Similar positive results have been reported with behavioral therapy in another study. ${ }^{100}$ Baclofen has also been applied with success in a small open label study. ${ }^{101}$

\section{Medical and surgical treatment in NERD, hypersensitive esophagus and functional heartburn}

Patients presenting with symptoms suggestive of reflux disease are often empirically treated with lifestyle advices and acid suppressive drugs, including PPIs. ${ }^{102-105}$ When patients do not respond to standard therapy, endoscopic and functional testing is performed to challenge the initial diagnosis and to investigate the reasons of treatment refractoriness. While the presence of erosive esophagitis confirms the diagnosis, a negative endoscopy cannot be used to rule out reflux disease, as a substantial part of GERD patients do not have any abnormalities seen on endoscopy (NERD). ${ }^{106,107}$

In most clinical trials, NERD patients are defined only by the presence of typical reflux symptoms and negative endoscopy. However, without appropriate functional testing it is difficult, if not impossible, to distinguish between FH, functional dyspepsia, and true NERD. Thus, the heterogeneity of the trial participants across studies could cause underestimation of the response rates to PPI treatment in NERD. Indeed, in a recent meta-analysis, Weijenborg et al. observed that 
in well-defined NERD patients (diagnosed by means of endoscopy and pathophysiological tests), the estimated complete symptom response rate after PPI therapy is comparable to the response rate in patients with ERD. In this paper the authors concluded that the previously reported low response rate in studies with patients classified as NERD was likely the result of inclusion of patients with upper gastrointestinal symptoms that did not have reflux disease. ${ }^{108}$

Previously, Fass et al. ${ }^{109}$ observed a direct and strong correlation between acid exposure time and the positive response rate to omeprazole (40 $\mathrm{mg}$ in the morning and $20 \mathrm{mg}$ in the evening).

Zerbib et al. ${ }^{52}$ described that patients with either positive symptom-reflux association analysis or AET $>5 \%$ were more frequently associated with a positive response to PPI therapy. However, the main finding of this study was that performing the multivariate analysis, the only factors associated with inadequate response to PPI were BMI $\leq 25 \mathrm{~kg} / \mathrm{m}^{2}$ and the presence of functional dyspepsia or irritable bowel syndrome symptoms. Patel et al. ${ }^{110}$ observed that only acidbased reflux parameters (total AET and AET $>4.0 \%$ ) offer greater value over impedance-based nonacid-reflux parameters (total reflux events and bolus exposure time) in predicting symptomatic responses to PPI therapy.

Controlling heartburn in patients with NERD can also be achieved with antacid or alginate compounds. Many clinical trials have demonstrated the benefit of these drugs, which continue to have a role in quickly relieving typical reflux symptoms in both NERD and erosive reflux disease. ${ }^{11-117}$ However, these over-the-counter drugs need multiple doses during the day, because of their short duration of action. Sodium alginate is a polysaccharide derived from seaweed. It binds water to form a viscous gum that floats in the proximal stomach, thereby separating the acid pocket from the distal esophagus. ${ }^{118}$ Some commercially available alginate preparations also contain an antacid. Sodium alginate might have the theoretical advantage of blocking both acid and WARs on the basis of the mechanical formation of a raft floating above gastric secretions, but its effect on the latter kind of reflux was not confirmed in a study using MII-pH testing. ${ }^{119}$ As above-mentioned, most reflux episodes happen during TLESRs, and these can be inhibited pharmacologically. The $\gamma$ - 
aminobutyric acid (GABA)B-receptor agonist baclofen reduces the incidence of TLESRs and reflux episodes. Vela et al. ${ }^{120}$ have shown that baclofen induces a reduction of total amount of reflux events and contributes to improve the symptoms complained of by patients with reflux. On the other hand, this drug is not suitable for treatment of GERD because of its mainly neurologic central side-effects. ${ }^{121}$ Unfortunately, the development of new drugs of this type with less severe adverse events than baclofen has been stopped, because of poor efficacy. ${ }^{122}$

An additional therapeutic option might be surgery that should be considered for patients with proven GERD and for those patients with weakly acidic reflux events. ${ }^{16,123,124}$

Five-year results of a randomised European trial comparing maintenance PPI treatment (esomeprazole) with laparoscopic Nissen fundoplication ${ }^{125}$ showed that the remission rate did not differ between the two therapeutic strategies. However, at 5 years, acid regurgitation was more prevalent in the PPI group than in the fundoplication group.

Several uncontrolled trials have shown that fundoplication is able to control symptoms related to both acid and WARs ${ }^{16,41,54,126,127}$ In particular, Broeders et al. ${ }^{126}$ have demonstrated that patients with normal AET and positive symptom association (HE patients) might benefit from fundoplication as well as patients with abnormal AET, although an important limitation of this study should be reported in that about $40 \%$ of the HE patients had prior evidence of erosive esophagitis at endoscopy (i.e. not affected by HE per definition). Similarly, Patel et al. ${ }^{128}$ showed that anti-reflux medical and surgical therapy may improve symptoms in hypersensitive patients, in well-defined settings. Bredenoord et al. ${ }^{129}$ confirmed that fundoplication is able to reduce the abnormal levels of both chemical types of reflux. Patel et al. ${ }^{130}$ demonstrated in a large series of patients, after a 40-month follow-up period, that the response to laparoscopic anti-reflux therapy was consistent in patients selected by means of a MII-pH performed off-therapy. The authors showed that abnormal AET and the symptom-reflux association SAP consistently predicted symptomatic outcome in a multivariate analysis. 
Few data are available regarding characteristics of the refluxate, such as the presence of pepsin and bile acids that may contribute to symptom perception. ${ }^{131}$ In this review we focused that WARs events can cause not only regurgitation but also heartburn 5,71 and patients with symptomatic non-acid (weakly acidic) reflux on PPI treatment should be considered good candidates for anti-reflux surgery.

\section{Conclusions}

Gastroesophageal reflux disease is a very common condition. Heartburn and regurgitation are the symptoms of the typical reflux syndrome. Reflux characteristics, other than acidity, such as the presence of WARs and presence of pepsin may also contribute to symptom perception. The cornerstone for treatment of GERD-related symptoms is acid suppression with PPIs. In tertiary care center it is more frequent running into patients unresponsive to acid suppressive treatment. The lack of response to a sustained acid inhibition suggests that the symptoms are not due to reflux or, alternatively, that they are reflux-related but PPI-unresponsive. Pathophysiological diagnosis of GERD should be performed preferably by means of combined $\mathrm{pH}$-impedance measurement. Patients should be recommended to accurately record symptoms to obtain the best information from symptom-reflux correlation scores. Up and coming parameters obtained from impedance and $\mathrm{pH}$ tracings as well as the presence of gas, aerophagia and supragastric belching should be considered, particularly when patients fail to record symptoms before considering functional diagnosis. Future studies are needed on these topics.

When NERD patients have been well characterized, PPI treatment as well as antireflux surgery can be considered effective in them. It has been shown that a proportion of patients with reflux hypersensitivity can improve with antireflux surgery, especially if regurgitation is the main symptom and some structural disruption at the esophago-gastric junction may be documented. ${ }^{132-134}$. At present functional upper GI symptoms are an exclusion criterion for antireflux surgery. 
Aknowledgements:

We would like to thank Michio Hongo, Ram Dickman, Samuel Nurko, Sutep Gonlachanvit, Jerzy Sarosiek, Varocha Mahacha, and Roger P. Tatum for their contribution.

\section{Conflicts of interest}

The authors declare no conflicts of interest. 


\section{REFERENCES}

1. El-Serag, H.B., S. Sweet, C.C. Winchester, et al. 2014. Update on the epidemiology of gastrooesophageal reflux disease: a systematic review. Gut. 63: 871-880.

2. Vakil, N., S.V. van Zanten, P. Kahrilas, et al. 2006. The Montreal definition and classification of gastroesophageal reflux disease: a global evidence-based consensus. The American journal of gastroenterology. 101: 1900-1920; quiz 1943.

3. Modlin, I.M., R.H. Hunt, P. Malfertheiner, et al. 2009. Diagnosis and management of non-erosive reflux disease--the Vevey NERD Consensus Group. Digestion. 80: 74-88.

4. Savarino, E., P. Zentilin \& V. Savarino. 2013. NERD: an umbrella term including heterogeneous subpopulations. Nature reviews. Gastroenterology \& hepatology. 10: 371-380.

5. Fass, R. \& D. Sifrim. 2009. Management of heartburn not responding to proton pump inhibitors. Gut. 58: 295-309.

6. Sifrim, D. \& F. Zerbib. 2012. Diagnosis and management of patients with reflux symptoms refractory to proton pump inhibitors. Gut. 61: 1340-1354.

7. Aziz, Q., R. Fass, C.P. Gyawali, et al. 2016. Functional Esophageal Disorders. Gastroenterology.

8. Savarino, E., P. Zentilin, R. Tutuian, et al. 2008. The role of nonacid reflux in NERD: lessons

learned from impedance-pH monitoring in 150 patients off therapy. Am J Gastroenterol. 103: 26852693.

9. Savarino, E., P. Zentilin, R. Tutuian, et al. 2012. Impedance-pH reflux patterns can differentiate non-erosive reflux disease from functional heartburn patients. Journal of Gastroenterology. 47: 159168.

10. Savarino, E., E. Marabotto, P. Zentilin, et al. 2011. The added value of impedance-pH monitoring to Rome III criteria in distinguishing functional heartburn from non-erosive reflux disease. Dig Liver

Dis.

11. Hemmink, G.J., A.J. Bredenoord, B.L. Weusten, et al. 2008. Esophageal pH-impedance monitoring in patients with therapy-resistant reflux symptoms: 'on' or 'off' proton pump inhibitor? Am J Gastroenterol. 103: 2446-2453.

12. Zerbib, F., S. Roman, A. Ropert, et al. 2006. Esophageal pH-impedance monitoring and symptom analysis in GERD: a study in patients off and on therapy. Am J Gastroenterol. 101: 1956-1963.

13. Viazis, N., A. Keyoglou, A.K. Kanellopoulos, et al. 2011. Selective Serotonin Reuptake Inhibitors for the Treatment of Hypersensitive Esophagus: A Randomized, Double-Blind, Placebo-Controlled Study. Am J Gastroenterol.

14. Giacchino, M., V. Savarino \& E. Savarino. 2013. Distinction between patients with non-erosive reflux disease and functional heartburn. Ann Gastroenterol. 26: 283-289.

15. Mainie, I., R. Tutuian, S. Shay, et al. 2006. Acid and non-acid reflux in patients with persistent symptoms despite acid suppressive therapy: a multicentre study using combined ambulatory impedance-pH monitoring. Gut. 55: 1398-1402.

16. Frazzoni, M., M. Piccoli, R. Conigliaro, et al. 2013. Refractory gastroesophageal reflux disease as diagnosed by impedance-pH monitoring can be cured by laparoscopic fundoplication. Surgical Endoscopy.

17. Dean, B.B., A.D. Gano, Jr., K. Knight, et al. 2004. Effectiveness of proton pump inhibitors in nonerosive reflux disease. Clin Gastroenterol Hepatol. 2: 656-664.

18. Numans, M.E., J. Lau, N.J. de Wit, et al. 2004. Short-term treatment with proton-pump inhibitors as a test for gastroesophageal reflux disease: a meta-analysis of diagnostic test characteristics. Ann Intern Med. 140: 518-527.

19. Dent, J., N. Vakil, R. Jones, et al. 2010. Accuracy of the diagnosis of GORD by questionnaire, physicians and a trial of proton pump inhibitor treatment: the Diamond Study. Gut. 59: 714-721.

20. Bytzer, P., R. Jones, N. Vakil, et al. 2012. Limited ability of the proton-pump inhibitor test to identify patients with gastroesophageal reflux disease. Clinical gastroenterology and hepatology: the official clinical practice journal of the American Gastroenterological Association. 10: 1360-1366.

21. Saleh, C.M., A.J. Smout \& A.J. Bredenoord. 2015. The diagnosis of gastro-esophageal reflux disease cannot be made with barium esophagograms. Neurogastroenterol Motil. 27: 195-200. 
22. Jonasson, C., B. Wernersson, D.A. Hoff, et al. 2013. Validation of the GerdQ questionnaire for the diagnosis of gastro-oesophageal reflux disease. Alimentary pharmacology \& therapeutics. 37: 564-572.

23. Lacy, B.E., R. Chehade \& M.D. Crowell. 2011. A prospective study to compare a symptom-based reflux disease questionnaire to 48-h wireless $\mathrm{pH}$ monitoring for the identification of gastroesophageal reflux (revised 2-26-11). Am J Gastroenterol. 106: 1604-1611.

24. Hershcovici, T. \& J. Zimmerman. 2008. Functional heartburn vs. non-erosive reflux disease: similarities and differences. Aliment Pharmacol Ther. 27: 1103-1109.

25. Park, E.Y., M.G. Choi, M. Baeg, et al. 2013. The value of early wireless esophageal pH monitoring in diagnosing functional heartburn in refractory gastroesophageal reflux disease. Dig Dis Sci. 58: 29332939.

26. Shapiro, M., C. Green, J.M. Bautista, et al. 2006. Functional heartburn patients demonstrate traits of functional bowel disorder but lack a uniform increase of chemoreceptor sensitivity to acid. $A m$ J Gastroenterol. 101: 1084-1091.

27. Savarino, E., P. Zentilin, E. Marabotto, et al. 2011. Overweight is a risk factor for both erosive and non-erosive reflux disease. Digestive and liver disease : official journal of the Italian Society of Gastroenterology and the Italian Association for the Study of the Liver. 43: 940-945.

28. Viazis, N., G.P. Karamanolis, J. Anastasiou, et al. 2013. Refractory GERD: increased body mass index is associated with persisting acid exposure but not hypersensitive esophagus or functional heartburn. Eur J Gastroenterol Hepatol. 25: 1450-1455.

29. Tolone, S., E. Savarino, N. de Bortoli, et al. 2016. Esophagogastric junction morphology assessment by high resolution manometry in obese patients candidate to bariatric surgery. International journal of surgery. 28 Suppl 1: S109-113.

30. Savarino, E., D. Pohl, P. Zentilin, et al. 2009. Functional heartburn has more in common with functional dyspepsia than with non-erosive reflux disease. Gut. 58: 1185-1191.

31. Savarino, V., E. Savarino, A. Parodi, et al. 2007. Functional heartburn and non-erosive reflux disease. Dig Dis. 25: 172-174.

32. de Bortoli, N., I. Martinucci, M. Bellini, et al. 2013. Overlap of functional heartburn and gastroesophageal reflux disease with irritable bowel syndrome. World journal of gastroenterology : WJG. 19: 5787-5797.

33. de Bortoli, N., I. Martinucci, E. Savarino, et al. 2014. Proton pump inhibitor responders who are not confirmed as GERD patients with impedance and $\mathrm{pH}$ monitoring: who are they?

Neurogastroenterology and motility : the official journal of the European Gastrointestinal Motility Society. 26: 28-35.

34. Savarino, V., E. Savarino \& P. Dulbecco. 2006. Proton pump inhibitor failure: why does it occur and how can it be managed? Digestion. 73: 215-217.

35. Kahrilas, P.J., G. Boeckxstaens \& A.J. Smout. 2013. Management of the patient with incomplete response to PPI therapy. Best Pract Res Clin Gastroenterol. 27: 401-414.

36. Pandolfino, J.E. \& M.A. Kwiatek. 2008. Use and utility of the Bravo pH capsule. J Clin Gastroenterol. 42: 571-578.

37. Sifrim, D., D. Castell, J. Dent, et al. 2004. Gastro-oesophageal reflux monitoring: review and consensus report on detection and definitions of acid, non-acid, and gas reflux. Gut. 53: 1024-1031.

38. Zentilin, P., E. Iiritano, P. Dulbecco, et al. 2006. Normal values of 24-h ambulatory intraluminal impedance combined with pH-metry in subjects eating a Mediterranean diet. Dig Liver Dis. 38: 226232.

39. Frazzoni, M., E. Savarino, M. Manno, et al. 2009. Reflux patterns in patients with short-segment Barrett's oesophagus: a study using impedance-pH monitoring off and on proton pump inhibitor therapy. Aliment Pharmacol Ther. 30: 508-515.

40. Boeckxstaens, G.E. \& A. Smout. 2010. Systematic review: role of acid, weakly acidic and weakly alkaline reflux in gastro-oesophageal reflux disease. Alimentary pharmacology \& therapeutics. 32: 334343.

41. Mainie, I., R. Tutuian, A. Agrawal, et al. 2006. Combined multichannel intraluminal impedance$\mathrm{pH}$ monitoring to select patients with persistent gastro-oesophageal reflux for laparoscopic Nissen fundoplication. Br J Surg. 93: 1483-1487. 
42. Savarino, E., P. Zentilin, M. Frazzoni, et al. 2010. Characteristics of gastro-esophageal reflux episodes in Barrett's esophagus, erosive esophagitis and healthy volunteers. Neurogastroenterology and motility : the official journal of the European Gastrointestinal Motility Society. 22: 1061-e1280.

43. Zentilin, P., P. Dulbecco, E. Savarino, et al. 2004. Combined multichannel intraluminal impedance and pH-metry: a novel technique to improve detection of gastro-oesophageal reflux literature review. Digestive and liver disease : official journal of the Italian Society of Gastroenterology and the Italian Association for the Study of the Liver. 36: 565-569.

44. Pandolfino, J.E. \& M.F. Vela. 2009. Esophageal-reflux monitoring. Gastrointest Endosc. 69: 917930, 930 e911.

45. Hershcovici, T., C.S. Wendel \& R. Fass. 2011. Symptom indexes in refractory gastroesophageal reflux disease: overrated or misunderstood? Clinical gastroenterology and hepatology : the official clinical practice journal of the American Gastroenterological Association. 9: 816-817.

46. Slaughter, J.C., M. Goutte, J.A. Rymer, et al. 2011. Caution about overinterpretation of symptom indexes in reflux monitoring for refractory gastroesophageal reflux disease. Clinical gastroenterology and hepatology : the official clinical practice journal of the American Gastroenterological Association. 9: 868-874.

47. Frazzoni, M., R. Manta, V.G. Mirante, et al. 2013. Esophageal chemical clearance is impaired in gastro-esophageal reflux disease--a 24-h impedance-pH monitoring assessment.

Neurogastroenterology and motility : the official journal of the European Gastrointestinal Motility Society. 25: 399-406, e295.

48. Frazzoni, M., E. Savarino, N. de Bortoli, et al. 2015. Analyses of the Post-reflux Swallow-induced Peristaltic Wave Index and Nocturnal Baseline Impedance Parameters Increase the Diagnostic Yield of Patients with Reflux Disease. Clinical gastroenterology and hepatology : the official clinical practice journal of the American Gastroenterological Association.

49. Savarino, E., R. Tutuian, P. Zentilin, et al. 2010. Characteristics of reflux episodes and symptom association in patients with erosive esophagitis and nonerosive reflux disease: study using combined impedance-pH off therapy. The American journal of gastroenterology. 105: 1053-1061.

50. Bredenoord, A.J. \& A.J. Smout. 2013. Association between reflux and symptoms during ambulatory reflux monitoring: pros and cons of existing methods. Neurogastroenterol Motil. 25: 633637.

51. Martinucci, I., N. de Bortoli, E. Savarino, et al. 2014. Esophageal baseline impedance levels in patients with pathophysiological characteristics of functional heartburn. Neurogastroenterology and motility : the official journal of the European Gastrointestinal Motility Society. 26: 546-555.

52. Zerbib, F., K. Belhocine, M. Simon, et al. 2012. Clinical, but not oesophageal pH-impedance, profiles predict response to proton pump inhibitors in gastro-oesophageal reflux disease. Gut. 61: 501506.

53. Farre, R., K. Blondeau, D. Clement, et al. 2011. Evaluation of oesophageal mucosa integrity by the intraluminal impedance technique. Gut. 60: 885-892.

54. Frazzoni, M., R. Conigliaro \& G. Melotti. 2011. Reflux parameters as modified by laparoscopic fundoplication in 40 patients with heartburn/regurgitation persisting despite PPI therapy: a study using impedance-pH monitoring. Dig Dis Sci. 56: 1099-1106.

55. Kessing, B.F., A.J. Bredenoord, P.W. Weijenborg, et al. 2011. Esophageal acid exposure decreases intraluminal baseline impedance levels. The American journal of gastroenterology. 106: 2093-2097.

56. de Bortoli, N., I. Martinucci, E. Savarino, et al. 2014. Association Between Baseline Impedance Values and Response Proton Pump Inhibitorsin Patients With Heartburn. Clinical gastroenterology and hepatology: the official clinical practice journal of the American Gastroenterological Association.

57. Gyawali, C.P. 2015. Redeeming Clinical Value of Esophageal pH Impedance Monitoring. Clin Gastroenterol Hepatol.

58. de Bortoli, N., I. Martinucci, E.V. Savarino, et al. 2015. Manually calculated oesophageal bolus clearance time increases in parallel with reflux severity at impedance-pH monitoring. Dig Liver Dis. 47: 1027-1032.

59. Woodland, P., M. Al-Zinaty, E. Yazaki, et al. 2012. In vivo evaluation of acid-induced changes in oesophageal mucosa integrity and sensitivity in non-erosive reflux disease. Gut. 
60. Koukias, N., P. Woodland, E. Yazaki, et al. 2015. Supragastric Belching: Prevalence and Association With Gastroesophageal Reflux Disease and Esophageal Hypomotility. J Neurogastroenterol Motil. 21: 398-403.

61. Tutuian, R., I. Mainie, A. Agrawal, et al. 2006. Nonacid reflux in patients with chronic cough on acid-suppressive therapy. Chest. 130: 386-391.

62. Ribolsi, M., E. Savarino, N. De Bortoli, et al. 2014. Reflux pattern and role of impedance-pH variables in predicting PPI response in patients with suspected GERD-related chronic cough. Alimentary pharmacology \& therapeutics. 40: 966-973.

63. Savarino, E., R. Carbone, E. Marabotto, et al. 2013. Gastro-oesophageal reflux and gastric aspiration in idiopathic pulmonary fibrosis patients. Eur Respir J.

64. Savarino, E., M. Bazzica, P. Zentilin, et al. 2009. Gastroesophageal reflux and pulmonary fibrosis in scleroderma: a study using $\mathrm{pH}$-impedance monitoring. American journal of respiratory and critical care medicine. 179: 408-413.

65. Blondeau, K., L.J. Dupont, V. Mertens, et al. 2008. Gastro-oesophageal reflux and aspiration of gastric contents in adult patients with cystic fibrosis. Gut. 57: 1049-1055.

66. Blondeau, K., L.J. Dupont, V. Mertens, et al. 2007. Improved diagnosis of gastro-oesophageal reflux in patients with unexplained chronic cough. Alimentary pharmacology \& therapeutics. 25: 723732.

67. Sifrim, D., L. Dupont, K. Blondeau, et al. 2005. Weakly acidic reflux in patients with chronic unexplained cough during 24 hour pressure, pH, and impedance monitoring. Gut. 54: 449-454.

68. Tack, J., G. Koek, I. Demedts, et al. 2004. Gastroesophageal reflux disease poorly responsive to single-dose proton pump inhibitors in patients without Barrett's esophagus: acid reflux, bile reflux, or both? Am J Gastroenterol. 99: 981-988.

69. Vela, M.F., L. Camacho-Lobato, R. Srinivasan, et al. 2001. Simultaneous intraesophageal impedance and $\mathrm{pH}$ measurement of acid and nonacid gastroesophageal reflux: effect of omeprazole. Gastroenterology. 120: 1599-1606.

70. Zerbib, F., A. Duriez, S. Roman, et al. 2008. Determinants of gastro-oesophageal reflux perception in patients with persistent symptoms despite proton pump inhibitors. Gut. 57: 156-160.

71. de Bortoli, N., I. Martinucci, E. Savarino, et al. 2014. Lower pH values of weakly acidic refluxes as determinants of heartburn perception in gastroesophageal reflux disease patients with normal esophageal acid exposure. Diseases of the esophagus : official journal of the International Society for Diseases of the Esophagus / I.S.D.E.

72. Tenca, A., S. Massironi, D. Pugliese, et al. 2015. Gastro-esophageal reflux and antisecretory drugs use among patients with chronic autoimmune atrophic gastritis: a study with $\mathrm{pH}$-impedance monitoring. Neurogastroenterol Motil.

73. Ribolsi, M., S. Emerenziani, T. Petitti, et al. 2012. Increased frequency and enhanced perception of reflux in non-erosive reflux disease patients non-responders to proton pump inhibitors. Digestive and liver disease : official journal of the Italian Society of Gastroenterology and the Italian Association for the Study of the Liver. 44: 549-554.

74. Farre, R., F. Fornari, K. Blondeau, et al. 2010. Acid and weakly acidic solutions impair mucosal integrity of distal exposed and proximal non-exposed human oesophagus. Gut. 59: 164-169.

75. Pearson, J.P., S. Parikh, R.C. Orlando, et al. 2011. Review article: reflux and its consequences-the laryngeal, pulmonary and oesophageal manifestations. Conference held in conjunction with the 9th International Symposium on Human Pepsin (ISHP) Kingston-upon-Hull, UK, 21-23 April 2010. Alimentary pharmacology \& therapeutics. 33 Suppl 1: 1-71.

76. Emerenziani, S., M. Ribolsi, M.P. Guarino, et al. 2014. Acid reflux episodes sensitize the esophagus to perception of weakly acidic and mixed reflux in non-erosive reflux disease patients. Neurogastroenterol Motil. 26: 108-114.

77. Emerenziani, S., D. Sifrim, F.I. Habib, et al. 2008. Presence of gas in the refluxate enhances reflux perception in non-erosive patients with physiological acid exposure of the oesophagus. Gut. 57: 443-447.

78. Frazzoni, M., H. Bertani, R. Manta, et al. 2014. Impairment of chemical clearance is relevant to the pathogenesis of refractory reflux oesophagitis. Dig Liver Dis. 46: 596-602.

79. Herregods, T.V., A.J. Bredenoord \& A.J. Smout. 2015. Pathophysiology of gastroesophageal reflux disease: new understanding in a new era. Neurogastroenterol Motil. 27: 1202-1213. 
80. Tobey, N.A., J.L. Carson, R.A. Alkiek, et al. 1996. Dilated intercellular spaces: a morphological feature of acid reflux--damaged human esophageal epithelium. Gastroenterology. 111: 1200-1205.

81. Katz, P.O., L.B. Gerson \& M.F. Vela. 2013. Guidelines for the diagnosis and management of gastroesophageal reflux disease. The American journal of gastroenterology. 108: 308-328; quiz 329. 82. Weijenborg, P.W., A.J. Smout, C. Verseijden, et al. 2014. Hypersensitivity to acid is associated with impaired esophageal mucosal integrity in patients with gastroesophageal reflux disease with and without esophagitis. Am J Physiol Gastrointest Liver Physiol. 307: G323-329.

83. van Malenstein, H., R. Farre \& D. Sifrim. 2008. Esophageal dilated intercellular spaces (DIS) and nonerosive reflux disease. Am J Gastroenterol. 103: 1021-1028.

84. Villanacci, V., P.G. Grigolato, R. Cestari, et al. 2001. Dilated intercellular spaces as markers of reflux disease: histology, semiquantitative score and morphometry upon light microscopy. Digestion. 64: 1-8.

85. Zentilin, P., V. Savarino, L. Mastracci, et al. 2005. Reassessment of the diagnostic value of histology in patients with GERD, using multiple biopsy sites and an appropriate control group. The American journal of gastroenterology. 100: 2299-2306.

86. Takubo, K., N. Honma, G. Aryal, et al. 2005. Is there a set of histologic changes that are invariably reflux associated? Archives of pathology \& laboratory medicine. 129: 159-163.

87. Barlow, W.J. \& R.C. Orlando. 2005. The pathogenesis of heartburn in nonerosive reflux disease: a unifying hypothesis. Gastroenterology. 128: 771-778.

88. Savarino, E., P. Zentilin, L. Mastracci, et al. 2013. Microscopic esophagitis distinguishes patients with non-erosive reflux disease from those with functional heartburn. Journal of Gastroenterology. 48: 473-482.

89. Fiocca, R., L. Mastracci, R. Riddell, et al. 2010. Development of consensus guidelines for the histologic recognition of microscopic esophagitis in patients with gastroesophageal reflux disease: the Esohisto project. Human pathology. 41: 223-231.

90. Kandulski, A., D. Jechorek, C. Caro, et al. 2013. Histomorphological differentiation of nonerosive reflux disease and functional heartburn in patients with PPI-refractory heartburn. Alimentary pharmacology \& therapeutics. 38: 643-651.

91. Kandulski, A., J. Weigt, C. Caro, et al. 2015. Esophageal intraluminal baseline impedance differentiates gastroesophageal reflux disease from functional heartburn. Clin Gastroenterol Hepatol. 13: $1075-1081$.

92. Pouderoux, P., G.A. Ergun, S. Lin, et al. 1996. Esophageal bolus transit imaged by ultrafast computerized tomography. Gastroenterology. 110: 1422-1428.

93. Bravi, I., P. Woodland, R.S. Gill, et al. 2013. Increased prandial air swallowing and postprandial gas-liquid reflux among patients refractory to proton pump inhibitor therapy. Clin Gastroenterol Hepatol. 11: 784-789.

94. Wyman, J.B., J. Dent, R. Heddle, et al. 1990. Control of belching by the lower oesophageal sphincter. Gut. 31: 639-646.

95. Bredenoord, A.J., B.L. Weusten, R. Timmer, et al. 2005. Relationships between air swallowing, intragastric air, belching and gastro-oesophageal reflux. Neurogastroenterology and motility : the official journal of the European Gastrointestinal Motility Society. 17: 341-347.

96. Zentilin, P., L. Accornero, P. Dulbecco, et al. 2005. Air swallowing can be responsible for nonresponse of heartburn to high-dose proton pump inhibitor. Dig Liver Dis. 37: 454-457.

97. Bredenoord, A.J. 2013. Management of belching, hiccups, and aerophagia. Clin Gastroenterol Hepatol. 11: 6-12.

98. Bredenoord, A.J., B.L. Weusten, D. Sifrim, et al. 2004. Aerophagia, gastric, and supragastric belching: a study using intraluminal electrical impedance monitoring. Gut. 53: 1561-1565.

99. Hemmink, G.J., L. Ten Cate, A.J. Bredenoord, et al. 2010. Speech therapy in patients with excessive supragastric belching--a pilot study. Neurogastroenterol Motil. 22: 24-28, e22-23.

100. Katzka, D.A. 2013. Simple office-based behavioral approach to patients with chronic belching. Dis Esophagus. 26: 570-573.

101. Blondeau, K., V. Boecxstaens, N. Rommel, et al. 2012. Baclofen improves symptoms and reduces postprandial flow events in patients with rumination and supragastric belching. Clin Gastroenterol Hepatol. 10: 379-384. 
102. van Pinxteren, B., K.E. Sigterman, P. Bonis, et al. 2010. Short-term treatment with proton pump inhibitors, H2-receptor antagonists and prokinetics for gastro-oesophageal reflux disease-like symptoms and endoscopy negative reflux disease. Cochrane Database Syst Rev. CD002095. 103. Ness-Jensen, E., K. Hveem, H. El-Serag, et al. 2015. Lifestyle intervention in gastroesophageal reflux disease. Clinical gastroenterology and hepatology : the official clinical practice journal of the American Gastroenterological Association.

104. de Bortoli, N., G. Guidi, I. Martinucci, et al. 2016. Voluntary and controlled weight loss can reduce symptoms and proton pump inhibitor use and dosage in patients with gastroesophageal reflux disease: a comparative study. Dis Esophagus. 29: 197-204.

105. De Bortoli, N., S. Tolone \& E.V. Savarino. 2015. Weight Loss Is Truly Effective in Reducing Symptoms and Proton Pump Inhibitor Use in Patients With Gastroesophageal Reflux Disease. Clin Gastroenterol Hepatol. 13: 2023.

106. Ronkainen, J., P. Aro, T. Storskrubb, et al. 2005. High prevalence of gastroesophageal reflux symptoms and esophagitis with or without symptoms in the general adult Swedish population: a Kalixanda study report. Scand J Gastroenterol. 40: 275-285.

107. Zagari, R.M., L. Fuccio, M.A. Wallander, et al. 2008. Gastro-oesophageal reflux symptoms, oesophagitis and Barrett's oesophagus in the general population: the Loiano-Monghidoro study. Gut. 57: 1354-1359.

108. Weijenborg, P.W., F. Cremonini, A.J. Smout, et al. 2012. PPI therapy is equally effective in welldefined non-erosive reflux disease and in reflux esophagitis: a meta-analysis. Neurogastroenterology and motility : the official journal of the European Gastrointestinal Motility Society. 24: 747-757, e350.

109. Fass, R., J.J. Ofman, R.E. Sampliner, et al. 2000. The omeprazole test is as sensitive as 24-h oesophageal $\mathrm{pH}$ monitoring in diagnosing gastro-oesophageal reflux disease in symptomatic patients with erosive oesophagitis. Aliment Pharmacol Ther. 14: 389-396.

110. Patel, A., G.S. Sayuk \& C.P. Gyawali. 2014. Acid-based parameters on pH-impedance testing predict symptom improvement with medical management better than impedance parameters. Am J Gastroenterol. 109: 836-844.

111. Mandel, K.G., B.P. Daggy, D.A. Brodie, et al. 2000. Review article: alginate-raft formulations in the treatment of heartburn and acid reflux. Aliment Pharmacol Ther. 14: 669-690.

112. Giannini, E.G., P. Zentilin, P. Dulbecco, et al. 2006. A comparison between sodium alginate and magaldrate anhydrous in the treatment of patients with gastroesophageal reflux symptoms. Dig Dis Sci. 51: 1904-1909.

113. Savarino, E., N. de Bortoli, P. Zentilin, et al. 2012. Alginate controls heartburn in patients with erosive and nonerosive reflux disease. World journal of gastroenterology: WJG. 18: 4371-4378.

114. Reimer, C., A.B. Lodrup, G. Smith, et al. 2016. Randomised clinical trial: alginate (Gaviscon Advance) vs. placebo as add-on therapy in reflux patients with inadequate response to a once daily proton pump inhibitor. Aliment Pharmacol Ther.

115. Thomas, E., A. Wade, G. Crawford, et al. 2014. Randomised clinical trial: relief of upper gastrointestinal symptoms by an acid pocket-targeting alginate-antacid (Gaviscon Double Action) - a double-blind, placebo-controlled, pilot study in gastro-oesophageal reflux disease. Aliment Pharmacol Ther. 39: 595-602.

116. Sweis, R., E. Kaufman, A. Anggiansah, et al. 2013. Post-prandial reflux suppression by a raftforming alginate (Gaviscon Advance) compared to a simple antacid documented by magnetic resonance imaging and $\mathrm{pH}$-impedance monitoring: mechanistic assessment in healthy volunteers and randomised, controlled, double-blind study in reflux patients. Aliment Pharmacol Ther. 37: 1093-1102. 117. De Ruigh, A., S. Roman, J. Chen, et al. 2014. Gaviscon Double Action Liquid (antacid \& alginate) is more effective than antacid in controlling post-prandial oesophageal acid exposure in GERD patients: a double-blind crossover study. Aliment Pharmacol Ther. 40: 531-537.

118. Kwiatek, M.A., S. Roman, A. Fareeduddin, et al. 2011. An alginate-antacid formulation (Gaviscon Double Action Liquid) can eliminate or displace the postprandial 'acid pocket' in symptomatic GERD patients. Aliment Pharmacol Ther. 34: 59-66.

119. Zentilin, P., P. Dulbecco, E. Savarino, et al. 2005. An evaluation of the antireflux properties of sodium alginate by means of combined multichannel intraluminal impedance and pH-metry.

Alimentary pharmacology \& therapeutics. 21: 29-34. 
120. Vela, M.F., R. Tutuian, P.O. Katz, et al. 2003. Baclofen decreases acid and non-acid post-prandial gastro-oesophageal reflux measured by combined multichannel intraluminal impedance and $\mathrm{pH}$.

Aliment Pharmacol Ther. 17: 243-251.

121. Lidums, I., A. Lehmann, H. Checklin, et al. 2000. Control of transient lower esophageal sphincter relaxations and reflux by the GABA(B) agonist baclofen in normal subjects. Gastroenterology. 118: 7-

13.

122. Boeckxstaens, G.E., H. Beaumont, J.G. Hatlebakk, et al. 2011. A novel reflux inhibitor lesogaberan (AZD3355) as add-on treatment in patients with GORD with persistent reflux symptoms despite proton pump inhibitor therapy: a randomised placebo-controlled trial. Gut. 60: 1182-1188.

123. Fuchs, K.H., B. Babic, W. Breithaupt, et al. 2014. EAES recommendations for the management of gastroesophageal reflux disease. Surgical Endoscopy. 28: 1753-1773.

124. Tolone, S., M. Furnari, N. de Bortoli, et al. 2015. Data on Symptom Association Analysis in Patients Undergoing Endoscopic Therapy Is Useful to Better Define a Successful Therapeutic Approach. Am J Gastroenterol. 110: 1621.

125. Galmiche, J.P., J. Hatlebakk, S. Attwood, et al. 2011. Laparoscopic antireflux surgery vs esomeprazole treatment for chronic GERD: the LOTUS randomized clinical trial. Jama. 305: 19691977.

126. Broeders, J.A., W.A. Draaisma, A.J. Bredenoord, et al. 2009. Oesophageal acid hypersensitivity is not a contraindication to Nissen fundoplication. Br J Surg. 96: 1023-1030.

127. Broeders, J.A., A.J. Bredenoord, E.J. Hazebroek, et al. 2011. Effects of anti-reflux surgery on weakly acidic reflux and belching. Gut. 60: 435-441.

128. Patel, A., G.S. Sayuk \& C.P. Gyawali. 2016. Prevalence, characteristics, and treatment outcomes of reflux hypersensitivity detected on pH-impedance monitoring. Neurogastroenterol Motil.

129. Bredenoord, A.J., W.A. Draaisma, B.L. Weusten, et al. 2008. Mechanisms of acid, weakly acidic and gas reflux after anti-reflux surgery. Gut. 57: 161-166.

130. Patel, A., G.S. Sayuk \& C.P. Gyawali. 2014. Parameters on Esophageal pH-Impedance Monitoring That Predict Outcomes of Patients With Gastroesophageal Reflux Disease. Clinical gastroenterology and hepatology: the official clinical practice journal of the American Gastroenterological Association.

131. Bredenoord, A.J. 2012. Mechanisms of reflux perception in gastroesophageal reflux disease: a review. Am J Gastroenterol. 107: 8-15.

132. Wang, D., A. Patel, M. Mello, et al. 2016. Esophagogastric junction contractile integral (EGJ-CI) quantifies changes in EGJ barrier function with surgical intervention. Neurogastroenterol Motil. 28: 639-646.

133. Tolone, S., N. De Bortoli, E. Marabotto, et al. 2015. Esophagogastric junction contractility for clinical assessment in patients with GERD: a real added value? Neurogastroenterol Motil. 27: 14231431.

134. Tolone, S., C. de Cassan, N. de Bortoli, et al. 2015. Esophagogastric junction morphology is associated with a positive impedance-pH monitoring in patients with GERD. Neurogastroenterol Motil. 27: $1175-1182$. 